\title{
Analysis of hospitalization expenditure and its influencing factors for lung cancer patients in China: a research based on the "System of Health Accounts 2011"
}

\section{Le Pan}

The First Hospital of China Medical University

Zhaolin Meng

China Medical University

\section{Yuanyi Cai}

China Medical University

Huazhang Wu( $\sim$ hzwu@cmu.edu.cn )

https://orcid.org/0000-0002-8761-2549

\section{Research article}

Keywords: Lung cancer, Hospitalization expenditure, Influencing factor

Posted Date: May 3rd, 2020

DOI: https://doi.org/10.21203/rs.3.rs-23264/v1

License: (c) (i) This work is licensed under a Creative Commons Attribution 4.0 International License.

Read Full License 


\section{Abstract}

Background At present, lung cancer is the common malignant tumor of respiratory system worldwide. The disease poses a serious health threat and a substantial economic burden to patients. The economic risks brought by lung cancer arouses widespread public concern. Therefore, the target of this research is to calculate lung cancer expenditure in China under the framework of the System of Health Account 2011 (SHA 2011). Intensive studies on working mechanism of influencing factors to lung cancer expenditure will be further explored, in order to achieve the goal of controlling lung cancer expenditure.

Methods A multistage stratified sampling method was conducted in Liaoning province in China, and a total of 23559 patients were included into hospitalization expenditure analysis according to the framework of SHA 2011. The relation between the total hospitalization expenditure of lung cancer and its influencing factors including of demographic characteristics, diagnosis and treatment, and hospital condition was analyzed with the multiple stepwise regression analysis. The impact mechanism of these influencing factors was revealed through path analysis and survival analysis.

Results The total hospitalization expenditure of lung cancer was $\$ 1581.8$. Multiple stepwise regression analysis indicated that the total hospitalization expenditure was associated with length of stay, surgery, hospital level, insurance status, and hospital type, according to the sequence of standardized estimate $(\beta)$. Length of stay contributed the most to the model R-square. Path analysis showed that surgery, hospital type, and insurance status not only made a direct impact on the hospitalization expenditure, but also made an indirect impact on it through the length of stay. Through survival analysis, we found selffunded patients of lung cancer were quicker to run out of the affordable money.

Conclusions Lung cancer brought a heavy economic burden for patients. More efficient and stringent clinical control strategies should be conducted to limit the increase of the expenditure.

\section{Background}

Lung cancer is the common malignant tumor of respiratory system, often occurs in bronchial epithelial cells, which poses a serious threat to human health worldwide. In 2018, approximately 18.079 million new cases of malignant tumor happened worldwide, among which new cases of lung cancer was 2.094 million, accounting for $11.58 \%$ of new cases of tumor across the world. The death cases of global malignant tumor were 9.555 million and the death of lung cancer was 1.761 million, taking up $18.43 \%$ of global tumor death cases, indicated that lung cancer was the most commonly diagnosed cancer, and the leading cause of cancer death [1]. In China, according to the newly updated data of National Cancer Center in 2019, lung cancer ranked top among national malignant tumors based on the sequence of patients' number. The estimated results showed that the new cases of lung cancer were 0.787 million in 2015 , with the incidence rate of $57.26 / 100,000$. According to the sequence of death number, lung cancer also ranked the first among the malignant tumors in China. The number of people died from lung cancer was 0.631 million in 2015 , with the mortality rate of $45.87 / 100,000$ [2]. 
With the occurrence of lung cancer, it brings a heavy economic burden to the society and family. The overall direct costs of caring for patients of lung cancer in Europe were amounting to more than $€ 3$ billion per year [3]. The developed countries, like England, Germany, France and Switzerland, the average period of patients' overall disease course was 19.8 months, with the direct medical cost of $€ 10,000-30,000$ per person [4]. Overall expenditures for treating early non-small cell lung cancer (NSCLC) ranged from \$ 24,040 to $\$ 97,774$ (Canadian Dollar) in 13 countries [5]. In China, a systematic review investigating lung cancer cost in Chinese population from database inception to September, 2017 through PubMed, Scopus, the Cochrane Library, Chinese National Knowledge Infrastructure (CNKI), Wan Fang Data, and VIP Data was conducted, and found the medical cost for the inpatient per-time in one year ranged from $¥ 8,582$ to $¥ 65,562[6]$.

On a global scope, although the researches on hospitalization expenditure and influencing factors of lung cancer have already been carried out, current studies on lung cancer expenditure lack the support of systematic accounting framework, decreasing the accuracy of expenditure calculation result and the comparability among different countries. In this research, the System of Health Accounts 2011 (SHA 2011) framework is introduced. SHA 2011 is revised and promoted by international organizations like OECD, EUROSTAT and WHO, the purpose of which is to establish a comprehensive and internal unified accounting system that can be compared across nations. SHA 2011 separates current health expenditure and capital formation from total health care expenditure. Three core dimensions, namely financing schemes, health care provider, and health care function are included. The key information in expanding dimensions like disease categories, age, sex, and region of health care consumers are included [7-8]. Therefore, under the framework of SHA 2011, we could conduct institutionalizing collection and analysis of the data of lung cancer, and were also able to accurately extract medical expenditure including drug, laboratory tests, surgery, etc, as well as the patients' demographic and clinical information like medical insurance, hospital type, etc.

Theses information could also provide a chance to analyze the key points associated with cost control. Therefore, the linear connection between influencing factors and lung cancer expenditure was explored in this research. We hypothesized that the mediating effect also existed, thus the further path analysis and survival analysis were carried out to analyze the indirect impact on the lung cancer expenditure of the influencing factors through intermediate variables.

Nowadays, China is facing the double burden of prevention and treatment for infectious disease and chronic disease. As one of the main chronic diseases, lung cancer not only threatens people's health, but also brings heavy economic loss to the whole society. On the premise of ensuring the quality for diagnosis and treatment, with scientific use of medical resources and effective control on treatment cost for lung cancer, the economic burden will be significantly reduced for the patients' family and the society. We hope that this research could systematically reveal the economic risks of lung cancer, meanwhile provides key points to the government and medical institutions for the prevention and treatment of lung cancer, and realizes reasonable intervention on the high treatment cost of lung cancer. 


\section{Methods}

\section{Data source}

According to the framework of SHA 2011, the expenditure data was collected from inpatients and outpatients of the sample medical institutions in Liaoning province, from 2015 to 2017. Liaoning

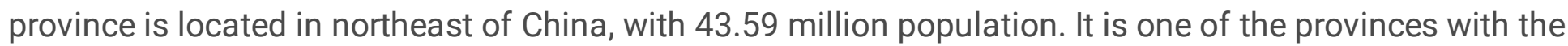
highest incidence rate of lung cancer in China. The incidence of lung cancer in Liaoning Province in 2015 was 78.5 / 100,000, which was higher than the incidence of lung cancer in the whole country [9].

\section{Study sample}

A multistage stratified sampling method was conducted in the field survey. The first stage was to select the sample areas. A total of 5 cities including Dalian, Fushun, Jinzhou, Tieling, and Panjin were selected, according to economic development standard and health service provision, etc. Then, 1 district and 2 counties were randomly selected in the above five cities. The second stage was to select the medical institutions. A total of 1227 medical institutions, including 83 public health institutions, 83 hospitals, 1061 grass-roots health care institutions, were selected, according to the type and level of institutions. Patients' information was collected to establish a database in these sample institutions, including age, sex, admission and discharge date, disease, International Classification of Disease, the 10th revision (ICD10), total expenditure, cost items, and insurance type, etc. The database included 5.2 million records in 2015, 5.3 million records in 2016, and 5.8 million records in 2017.

The cost data of lung cancer was extracted from the database. Patients with ICD-10 code 'C 34 ' were selected. Further screening was conducted, based on the following inclusion criteria: 1) inpatients in the institution type of hospital, 2) age $\geq 18$ years old. Through data cleaning of extreme and missing values, repeated data, logic errors, a total of 23559 valid records were included into final analysis.

\section{Assessment of hospitalization expenditure for patients}

The hospitalization expenditures and reimbursements for patients from the year 2015 to 2017 were calculated. This calculation included the total expenditure and nine specific categories: 1) test, 2) check, 3) diagnostics, 4) treatment, 5) surgery, 6) drugs 7) nursing, 8) bed fee, and 9) other (medical consumables, etc).

\section{Measurements of demographic characteristics, diagnosis and treatment, and hospital condition}

Demographic characteristics included age, sex, and insurance status. Insurance status was categorized as urban employees' basic medical insurance (UEBMI), urban residents' basic medical insurance (URBMI) and new rural cooperative medical system (NCMS), and self-funded, according to the sequence of reimbursement level. Diagnosis and treatment consisted of length of stay and surgery. Hospital condition was assessed based on 2 items: 1) hospital level, and 2) hospital type. Hospitals could be divided into 
provincial hospital $(\mathrm{PH})$, municipal hospital( $\mathrm{MH})$, and district and county hospital (DCH) according to its level, while provincial level representing the highest standard of hospitals in this area. Judging from hospital type, hospitals could be assessed into three unordered categories, namely general hospital (GH), special hospital (SH), and traditional Chinese medicine hospital (TCMH). Multiple regression model was conducted to analyze the impact on the total hospitalization expenditure of these influencing factors.

\section{Analysis of the impact mechanism of the factors}

Subsequent path analysis was implemented to explore the direct and indirect impact on the hospitalization expenditure for all the influencing factors. We established the path analysis diagram using multiple regression model, based on the following assumption.

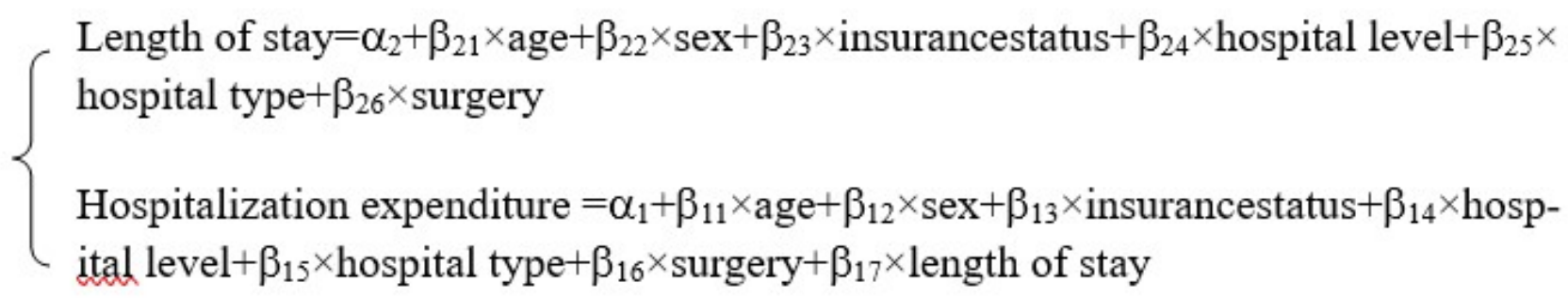

Furthermore, survival analysis was conducted to quantify the indirect impact on the expenditure of the influencing factors through length of stay, if assumption was proved. Patients met the criterion of end event were included. End event was defined as 'the catastrophic health payment occurred'. We studied how long it would last in different factor groups before end event occurred, and established survival plots. Catastrophic health payment was calculated according to the explanation recommended by WHO-'household health expenditures account for $40 \%$ or more of non-food consumption expenditures'.

\section{Statistical analysis}

The difference influencing factor groups in the univariate analysis was tested by the Wilcoxon rank sum test. A multiple stepwise regression analysis was used to clarify the influencing factors for the total hospitalization expenditure and length of stay. Variables significant at the 0.05 level in the univariate analysis were included in the model, and items with $P>0.10$ were eliminated. Dummy variables were constructed for the categorical variables such as insurance status, hospital level and hospital type. Moreover, Kaplan-Meier method was used to establish the Survival Analysis plot to explore the impact degree of these influencing factors. In the study, the logarithmic transition was conducted, because the total expenditure, nine specific cost items, and the length of stay were not normally distributed. The cost data were adjusted according to consumer price index (CPI), and the currency exchange rate used was US\$1 to $¥ 6.9364$. SPSS 21.0 (SPSS Inc., Chicago, IL, USA) was used for the statistical analyses.

\section{Results}




\section{Basic situation and composition of the hospitalization expenditure}

The total median expenditure for patients of lung cancer was $\$ 1581.8$, and that value was $\$ 1831.6$, $\$ 1862.9$, and $\$ 1165.1$, respectively during the year of 2015 to 2017. The out-of-pocket payment (OOP) for patients decreased from $\$ 782.4$ to $\$ 411.5$ in the three years (Table 1). As to the composition of the hospitalization expenditure, the proportion of drug expenditure was the highest among all the nine specific categories (Fig. 1), up to $41.6 \%$. Moreover, three main constituents could be extracted from the nine items, according to clinical practice. The contribution of diagnostics component (check, test, diagnostics), treatment component (treatment, drugs, surgery, other), and care component (bedfee, nursing) was $14.1 \%, 82.3 \%$, and $3.6 \%$, respectively (Fig. 2 ).

Table 1

Medical expenditure of lung cancer (\$)

\begin{tabular}{|c|c|c|c|}
\hline & Medical expenditure median (IQR) & $\begin{array}{l}\text { Reimbursement } \\
\text { median (IQR) }\end{array}$ & $\begin{array}{l}\text { OOP } \\
\text { Median (IQR) }\end{array}$ \\
\hline 2015 & $1831.6(1018.0,4014.5)$ & $705.3(184.5,1655.0)$ & $782.4(320.3,2347.2)$ \\
\hline 2016 & $1862.9(931.5,6457.7)$ & $724.9(0,2229.2)$ & $740.9(268.0,3661.7)$ \\
\hline 2017 & $1165.1(735.4,2141.9)$ & $667.4(313.0,1314.2)$ & $411.5(194.7,1068.7)$ \\
\hline Total & $1581.8(882.6,3562.1)$ & $691.7(203.9,1624.7)$ & $619.6(254.1,1928.2)$ \\
\hline
\end{tabular}

\section{Influencing factors of the hospitalization expenditure}

The characteristics and distributions of the total expenditure of lung cancer were shown in Table 2. There were significant differences in the age groups $(\mathrm{H}=33.868, \mathrm{P}<0.001)$, insurance status groups $(\mathrm{H}=$ 448.236, $P<0.001)$, hospital level groups $(H=3708.816, P<0.001)$, hospital type groups $(H=534.781, P<$ $0.001)$, length of stay groups $(H=9846.413, P<0.001)$, and surgery groups $\left(U=1.371 \times 10^{7}, P<0.001\right)$. 
Table 2

The characteristics and distributions of the total hospitalization expenditure

\begin{tabular}{|c|c|c|c|c|}
\hline Variables & & $\mathbf{n}$ & Median (IQR)\$ & $P$ \\
\hline \multirow[t]{4}{*}{ Age(yr.) } & $18 \llbracket 40$ & 330 & $1725.2(838.6,5309.7)$ & $<0.001$ \\
\hline & $41 \rrbracket 60$ & 8674 & $1540.6(850.0,3577.9)$ & \\
\hline & $61 \rrbracket 80$ & 13286 & $1620.0(910.7,3685.2)$ & \\
\hline & $\geq 81$ & 1269 & $1444.7(843.5,2751.7)$ & \\
\hline \multirow[t]{2}{*}{ Sex } & Female & 9796 & $1587.9(841.3,4122.0)$ & 0.917 \\
\hline & Male & 13763 & $1580.7(916.2,3292.1)$ & \\
\hline \multirow[t]{3}{*}{ Insurance status } & UEBMI & 12134 & 1769.3(976.6,4371.6) & $<0.001$ \\
\hline & URBMI and NCMS & 8316 & 1309.8(769.3,2602.1) & \\
\hline & SF & 3109 & 1607.5(917.7,4183.3) & \\
\hline \multirow[t]{3}{*}{ Hospital level } & $\mathrm{PH}$ & 16299 & $2103.0(1143.5,5527.2)$ & $<0.001$ \\
\hline & $\mathrm{MH}$ & 5598 & $999.9(640.6,1533.7)$ & \\
\hline & $\mathrm{DCH}$ & 1662 & $846.8(493.5,1331.3)$ & \\
\hline \multirow[t]{3}{*}{ Hospital Type } & $\mathrm{GH}$ & 17904 & 1513.3(841.5,3389.3) & $<0.001$ \\
\hline & $\mathrm{SH}$ & 3169 & $2369.4(1252.0,7388.0)$ & \\
\hline & $\mathrm{TCMH}$ & 2486 & $1336.9(916.9,2362.6)$ & \\
\hline \multirow[t]{4}{*}{ Length of stay (d) } & $1 \otimes 7$ & 6608 & 718.7(415.9,1124.2) & $<0.001$ \\
\hline & $8 \otimes 14$ & 9242 & $1540.2(1035.2,2673.6)$ & \\
\hline & $15 \rrbracket 21$ & 4765 & $2765.7(1617.6,7079.4)$ & \\
\hline & $\geq 22$ & 2944 & $5510.8(3093.2,9509.1)$ & \\
\hline \multirow[t]{2}{*}{ Surgery } & No & 17625 & $1263.2(750.6,2089.7)$ & $<0.001$ \\
\hline & Yes & 5934 & $7527.3(3005.8,9427.8)$ & \\
\hline
\end{tabular}

Multiple stepwise regression analysis was used to establish the regression equation. As the continuous variable, age was not correlated stably (positive or negative) with the total expenditure, and was excluded from the regression equation. Old age of lung cancer patients was not always accompanied with high payment. Actually, the previous study showed that the treatment expenditure of cancer was higher in younger patients than in older patients [10]. Thus, total of five factors including four categorical variables 
(insurance status, hospital level, hospital type, and surgery) and one continuous variable (length of stay) were selected $(F=5890.089, p<0.001)$. The total logarithmic expenditure of lung cancer was significantly related to logarithmic length of stay, surgery, hospital level, insurance status, and hospital type according to the sequence of the standardized estimate $(\beta)$. The contribution to the model R-square attributable to the five factors ( $\Delta$ R-square) was $0.47,0.143,0.039,0.006$, and 0.009 , respectively (Table 3 and Table 4 ). Collinearity diagnostics including of the method of eigenvalue, condition index, and variance proportion was conducted to analyze the multicollinearity among the variables and showed weak multiple collinearity among them.

Table 3

Multiple stepwise regression analysis for clarifying the major factors influencing the economic burden attributable to logarithmic hospitalization expenditure

\begin{tabular}{|llll|}
\hline Variables & $\begin{array}{l}\text { Parameter } \\
\text { estimate (B) }\end{array}$ & $\begin{array}{l}\text { Standardized } \\
\text { estimate (B) }\end{array}$ & Model R-Square \\
\hline Intercept & 1.503 & \\
\hline Ig Length of stay & $0.807^{*}$ & 0.536 \\
\hline Surgery (Yes vs. No) & $0.424^{*}$ & 0.382 \\
\hline Hospital level (PH vs. DCH) & $0.262^{*}$ & 0.251 \\
\hline Hospital level (MH vs. DCH) & $0.069^{*}$ & 0.061 \\
\hline Insurance status (UEBMI vs. SF) & $0.121^{*}$ & 0.126 \\
\hline Insurance status (URBMI and NCMS vs. SF) & $0.093^{*}$ & 0.092 & \\
\hline Hospital type (GH vs. TCMH) & $0.114^{*}$ & 0.102 & \\
\hline Hospital type (SH vs. TCMH) & $0.072^{*}$ & 0.051 & \\
\hline *p < 0.001 & & & 0.667 \\
\hline
\end{tabular}


Table 4

The contributions of factors to the model R-square
Diagnosis and treatment
hospital condition
Demographic
characteristics

Statistic

Ig Length of

stay

Surgery

Hospital

level

Hospital

type

Insurance status

$\begin{array}{llllll}\mathrm{R}^{2} & 0.47 & 0.613 & 0.652 & 0.661 & 0.667\end{array}$
$\Delta \mathrm{R}^{2}$
0.47
$0.143 \quad 0.039$
0.009
0.006

\section{Impact mechanism of the factors}

Path analysis showed the direct and indirect impact on the hospitalization expenditure for the variables. Surgery, hospital type, and insurance status not only made a direct impact on the hospitalization expenditure, but also made an indirect impact on it through the length of stay (Fig. 3).

Furthermore, survival analysis was conducted to quantify the indirect impact on the expenditure of the three influencing factors (surgery, hospital type, and insurance status) through length of stay. Catastrophic health payment was $\$ 720$, and we extended this value from $\$ 710$ to $\$ 730$. Thus, the OOP of 150 patients matched this criterion when they were discharged. The results of survival analysis were shown in Fig. 4. Self-funded patients were quicker to run out of the affordable money than patients with UEBMI or URBMI \&NCMS (Log Rank $\chi^{2}=28.232, P<0.001$ ). In addition, patients undergoing surgery could let the money maintain much more time, compared with patients with no surgery (Log Rank $\chi^{2}=2.028, P$ $=0.154$ ). As to the hospital type, patients in the general hospital were prone to reach the catastrophic health payment in a short time, but patients left sustained their money much longer ( $\log \operatorname{Rank} \chi^{2}=0.467$, $P=0.792)$.

\section{Discussion}

This study found that the median hospitalization expenditure of lung cancer patients was $\$ 1581.8$, higher than the level of $\$ 1281.7$ per capita for all the hospitalized patients in China [11]. Compared with the national per capita disposable income, the lung cancer patients' hospitalization expenditure occupied 
$32.4 \%$ of urban residents per capita disposable income, and $91.7 \%$ of the rural residents [12]. This shows that lung cancer will bring heavy burden to the entire society.

From the perspective of patients, the OOP decreased from $\$ 782.4$ to $\$ 411.5$ during the year of 2015 to 2017, which remarkably reduced the economic burden of lung cancer patients. However, it should be noted that among the study population, $61.8 \%$ of the patients were above 60 years old, retired with no income and subsisted on pension. In addition, the cost of treatment for malignant cancer is high. If complications like infection occurred during the course of treatment, patients' conditions would be aggravated and additional economic burden be increased [13]. Thus, the risk of illness and poverty for these patients still exist.

From the constituents of the medical cost, treatment component was the main part of the total hospitalization expenditure, and drug cost occupied a large proportion, up to $41.6 \%$. On one hand, high priced chemotherapy drugs and molecular target drugs are applied to lung cancer patients [14-15]. The high cost is also associated with lacking of domestic alternative medicines. On the other hand, China is entering a critical period of new round health care system reform, and compensation mechanism reform is an important part. The financial support to public hospitals by the government is relatively insufficient and public hospitals are running under pressure to a certain extent. Therefore, besides price factors, the compensation mechanism needs further improved. The problem of how to ensure all the medical institutions and physicians optimized the use of drugs is also needed to be solved. Currently, combined with other nations' reform experience, the Chinese government is proceeding a series of measure of reform. Zero-tariff policy on imported anti-cancer drugs and government's policy on pharmaceuticals purchase by public bidding are all included [16-17]. More anti-cancer drugs are brought into the medical insurance catalogue, as well as strengthening financial investment to health care institutions and implementing health technology assessment on drugs utilization.

The research showed that hospitalization expenditure was influenced by hospital length of stay, surgery, hospital type, hospital level and medical insurance situation. Hospital length of stay had the greatest impact on hospitalization expenditure and largest contribution to model R-Square. Since the severity of the disease, long time of observation and treatment for lung cancer hospitalized patients was needed, thereby the charges for beds, treatment and drugs increased accordingly. Moreover, for lacking of scientific and effective management on patients, some medical institutions increased the hospital stay and the cost in the process of treatment. The results of path analysis indicated that patients hospitalized cost was directly influenced by the stay length and indirectly by surgery, medical insurance situation and hospital type. The hospital length of stay was longer for those patients that had operations, medical insurance or having hospitalization in the special hospitals like oncology specialty hospitals. One reason was that compared with non-operative patients, the time for operative patients to recover was longer. For the patients with medical insurance, the usage of hospitalization service was increased to some extent compared with self-paid patients. The condition of lung cancer patients transferred from traditional Chinese medicine hospitals and general hospitals to specialized oncology hospitals was more severe. 
Therefore, reducing the hospital length of stay was the prime way to lower down patients' hospitalization expenditure. General control strategy should be established. Through clinical pathway management strategy, lung cancer patients could be scientifically and effectively treated and ineffective hospital length of stay be shortened, which had been demonstrated [18]. For various influencing factors, specific measures should be established to control the hospital length of stay. Through strict surgical indications and day surgery, hospital length of stay could be reduced [19]. By implementing diagnosis related groupsprospective payment system (DRGs-PPS) [20], the reform in UEBMI, URBMI and NCMS had been carried out. Meanwhile, hospitals' ability in lung cancer patients' diagnosis and treatment could be improved through coordination and cooperation.

In addition to hospital length of stay, patients whether or not taking operation was another direct influencing factor to hospitalized expenditure. The reason was that patients had to pay for the anesthesia drugs and the application of ECG monitoring and respirator cost, besides the operation cost. If complications like postoperative bleeding and atelectasis happened, additional cost would be paid. Therefore, physicians had to carefully choose the treatment plan. Currently, the treatment plan of lung cancer patients was based on pathological type and stage, chemotherapy, radiotherapy and operation were included [21]. Physicians had to select the most suitable treatment plan to patients based on their conditions.

Multiple linear regression showed that patients with medical insurance would pay higher hospitalized cost compared with the self-paid patients. Although the medical insurance system had provided more choices for patients, it should be aware that the moral hazard would appear and lead to overuse of medical resources between physicians and patients because of medical insurance [22]. Those with UEBMI had higher hospitalized cost than the patients with URBMI and NCMS. The reason was that China is still in the initial stage of developing basic medical insurance system. There was still a gap in financing level, the scope of insurance, the ratio of reimbursement among UEBMI, URBMI and NCMS. The lung cancer patients with UEBMI would have better medical guarantee and more choices of treatment plan. As to the self-paid patients, since the limitation of economy, some patients wouldn't go to the hospital until the disease advanced, therefore missed the best time to treat. Meanwhile, they were also faced with limited options concerning the treatment cost. The results of survival analysis showed that the hospitalized cost of self-paid patients was spent quicker and the risk of catastrophic expenditure was easier to appear. It indicated that the supervision of self-paid patients' hospitalized cost should be strengthened and more attentions should be paid from medical aid.

From the aspect of hospital type, lung cancer patients spent lower hospitalized cost in traditional Chinese medicine hospital. The reason was that traditional Chinese medicine and treatment could improve the physical function of the patients, prevent the recrudescence and reduce the post-operative complications. During the period of tumor chemotherapy and radiotherapy, traditional Chinese medicine could reduce its toxic side effects and enhance the therapeutic effect [23]. The introduction and combination of traditional Chinese medicine could make health care providers take patients as the foremost, focus on patients' life 
quality. It could help patients to set up an accurate view of health and life, learn to weight the advantage and disadvantage of the treatment and avoid the pain brought by excessive medical treatment [24].

Judging from the hospital level, lung cancer patients' hospitalization expenditure of district and county hospitals was obviously lower than the provincial and municipal hospitals. The charging rate and the complexity of patients' condition from high level hospitals was one important reason for the high hospitalized cost. Compared with the patients of acute symptom and the critical patients in the ICU, the price elasticity of demand existed in chronic disease such as lung cancer [25]. Price lever was useful in patients flow and reducing the burden of lung cancer patients hospitalized cost. The family physician system and hierarchical medical treatment system should be enhanced for patients' division. However, it required the lower level hospitals having strong support capacity and physicians meeting requirement of

treatment. Strengthening the construction of regional medical center and hospital groups like the Britain's hospital trust was the main way to upgrade the lower level hospitals capability.

There are two limitations in this study. First, more variables needed to be included. The data of this research was collected from sample medical institutions of Liaoning province, according to the framework of SHA 2011. Since the variables were limited, some variables like the patients' income and education level were not included, which would be the influencing factors of patients' hospitalization expenditure. Second, cross-sectional study was used in survival analysis and prospective research design was not included in the analysis of hospital length of stay for the patients reaching catastrophic expenditure rate. The patients whose self-paid cost reaching catastrophic expenditure rate but not discharge from hospitals were not included in the research and thereby reduced the sample size.

\section{Conclusions}

In the present study, we found lung cancer brought a heavy economic burden to patients under the framework of SHA 2011, especially for the patients in provincial and special hospitals, patients in more severe condition such as surgery and long hospitalization, and those without medical insurance, indicated that the government should pay more attention to the influencing factors and their interactions, conduct more efficient ways in order to relieve the economic risk for patients of lung cancer in China.

\section{Abbreviations}

SHA 2011: System of Health Accounts 2011; NSCLC: non-small cell lung cancer; CNKI: Chinese National Knowledge Infrastructure; ICD-10: International classification of disease, the 10th revision; UEBMI: Urban employees' basic medical insurance; URBMI: Urban residents' basic medical insurance; NCMS: New rural cooperative medical system; PH: Provincial hospital; MH: Municipal hospital; DCH: District and county hospital; GH: General hospital; SH: Special hospital; TCMH: Traditional Chinese medicine hospital; CPI: Consumer price index; OOP: Out-of-pocket payment; IQR: Interquartile range; DRGs-PPS: Diagnosis related groups-prospective payment system 


\section{Declarations}

\section{Ethics approval and consent to participate}

The Ethics Committee of China Medical University approved the study protocol. This analysis used secondary data, and the authors did not require direct contact with the participants, thus consent to participate was waived.

\section{Consent for publication}

Not applicable

\section{Availability of data and material}

The researchers who want to use the datasets in this study may contact the corresponding author for reasonable data request.

\section{Competing interests}

The authors have declared that no competing interests exist.

\section{Funding}

This work is funded by National Health Commission of the People's Republic of China. The funding body had no further involvement in the research process.

\section{Authors' contributions}

LP and ZIM studied the concept and made the whole design. HzW and YyC coordinated the data collection. LP conducted the analysis and interpretation of data. LP wrote the first draft. ZIM and YyC revised the manuscript.

\section{Acknowledgements}


The authors wish to thank the professors from the department of respiratory medicine for their insightful ideas and constructive suggestions on controlling lung cancer expenditure.

\section{References}

1.Bray F, Ferlay J, Soerjomataram I, Siegel RL, Torre LA, Jemal A. Global cancer statistics 2018: GLOBOCAN estimates of incidence and mortality worldwide for 36 cancers in 185 countries.CA Cancer J Clin. 2018; 68(6): 394-424.

2. Zheng RS, Sun KX, Zhang SW, Zeng HM, Zou XN, Chen R, et al. Report of cancer epidemiology in China, 2015. Chin J Oncol. 2019; 41(1): 19-28 .

3. ERS. The economic burden of lung disease. In: Gibson GJ, Loddenkemper R, Sibille Y, Sheffield LB, editors. European Lung White Book. United Kingdom: European Respiratory Society; 2013. p. 16-27.

4.Solem CT, Penrod JR, Lees M, Macahilig C, Luo L, VerlegerK et al. Cost drivers of lung cancer care: results from a retrospective chart review of pretreated advanced NSCLC patients in Europe. Value Health. 2017; 20(9): A431-A432.

5.Mahar AL, Fong R, Johnson A. The economic impact of treating early lung cancer: a systematic review. Value Health. 2011; 14(7): A440.

6. Song JF, Guan HJ, Liu GE. Direct medical cost of lung cancer in China: a systematic review. Chin J Evid-Based Med. 2019; 19 (1): 44-53.

7.OECD, Eurostat, WHO. A System of Health Accounts 2011edition. Paris:OECD; 2011.

8. Zhai TM, Zhang YH, Wan Q, Chai PP, Wei Q, Wang CC, et al. New System of Health Accounts Introduction of System of Health Accounts 2011. Chin Health Econ.2013; 32(1): 13-15.

9.Liaoning provincial center for disease control and prevention: Health and population status report of Liaoning province in 2015. http://www.Incdc.com/index.php?p=news_show\&p_id=17

1\&lanmu=17\&c_id=47. Accessed 26 Nov 2019.

10. Hwang I, Shin DW, Kang KH, Yang HK, Kim SY, Park JH, et al. Medical Costs and Healthcare Utilization among Cancer Decedents in the Last Year of Life in 2009. Cancer Res Treat. 2016; 48:365-75.

11. National Health Commission of the People's Republic of China. Statistical yearbook for health of China in 2018. Beijing: Peking Union Medical College Press; 2018. p. 113, 116.

12. National Bureau of Statistics of the People's Republic of China: Statistical bulletin of the national economy and social development in 2017. http://www.stats.gov.cn/tjsj/zxfb/201802/t2 
13. Dimick JB, Pelz RK, Consunji R, Swoboda SM, Hendrix CW, Lipsett PA. Increased resource use associated with catheter-related bloodstream infection in the surgical intensive care unit. Arch Surg. 2001; 136(2):229-34.

14. Thongprasert S, Permsuwan U. Crizotinib treatment for advanced non-small-cell lung cancer patients: a budget impact analysis based in Thailand. Curr Med Res Opin, 2017, 33(5): 955-961.

15. Warren JL, Yabroff KR, Meekins A, et al. Evaluation of trends in the cost of initial cancer treatment. J Natl Cancer Inst, 2008, 100(12): 888-897.

16. Busse R, Blümel M. Germany: Health system review. Health Systems in Transition.2014; 16(2):1-296.

17. Tan QL, Li WJ, Yang SY, Zhang WP, Wu GH, Chen JY. Study on the Affecting Factors and Optimizing Strategies of Drug Price in China on the Background of New Medical Reform. Chin J Pharm Econ. 2019; 14(9): 31-35, 45.

18. Cheah J. Clinical pathways--an evaluation of its impact on the quality of care in an acute care general hospital in Singapore. Singapore Medical Journal. 2000; 41(7): 335.

19. Marsden J, Lipp A, Kumar V. Day surgery: implications for general practice.Br J Gen Pract. 2016; 66(646):232-3.

20. Parekh TM, Bhatt SP, Westfall AO, Wells JM, Kirkpatrick D, lyer AS. Implications of DRG Classification in a Bundled Payment Initiative for COPD. Am J Accountable Care. 2017; 5(4): 12-18.

21. Xue C, Hu ZH, Jiang W, Zhao YY, Xu F, Huang Y, et al. National survey of the medical treatment status for non-small cell lung cancer (NSCLC) in China. Lung Cancer, 2012, 77(2): 371-375.

22. Thönnes S. Ex-post moral hazard in the health insurance market: empirical evidence from German data. Eur J Health Econ. 2019, 20 (9): 1317-1333.

23. Liu R, Hua BJ. Current Status and Analysis of Multidisciplinary Treatment Modalities with Traditional Chinese Medicine for Patients with Cancer. China Cancer.2014; 23(4)®311-315.

24. Hu AX, Pei ZY, Yan JJ, Zhao XL, Nan PH. Hospitalization expenditure and influencing factors of elderly patients with lung cancer. Chin J Gerontol. 2019; 39 (20): 5111-5113.

25. Folland S, Goodman AC, Stano M. The economics of health and health care. Beijing: China Renmin University Press; 2011. p. 191-3.

\section{Figures}




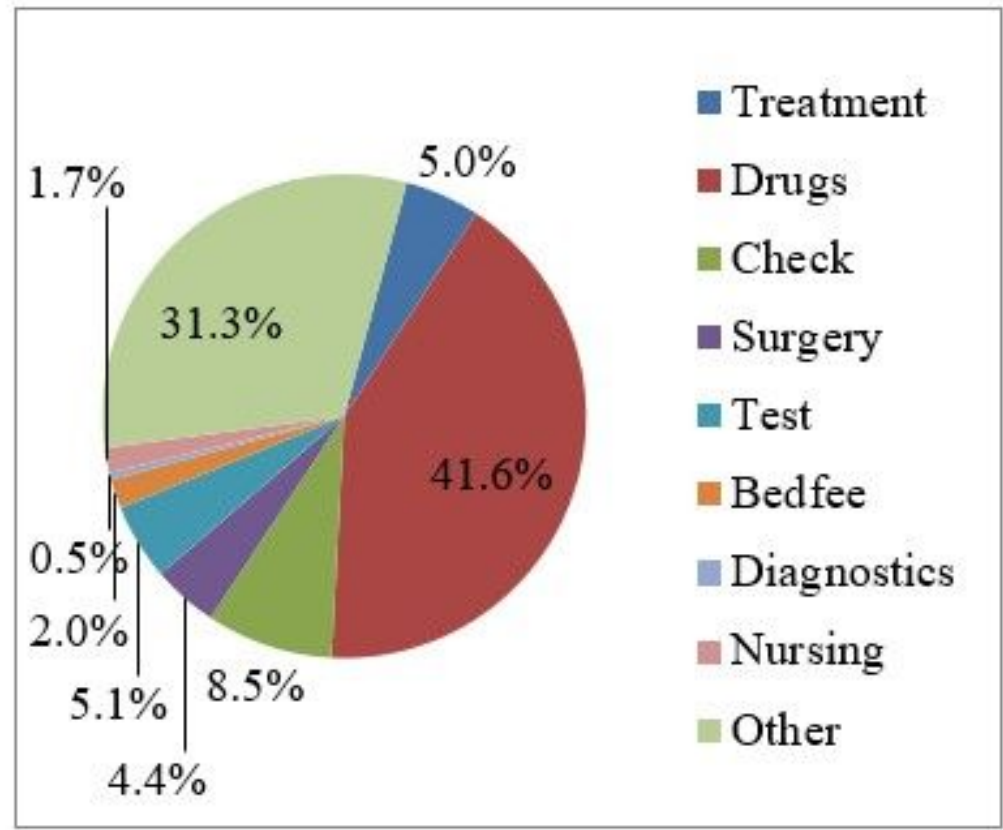

Figure 1

Proportion of hospitalization expenditure

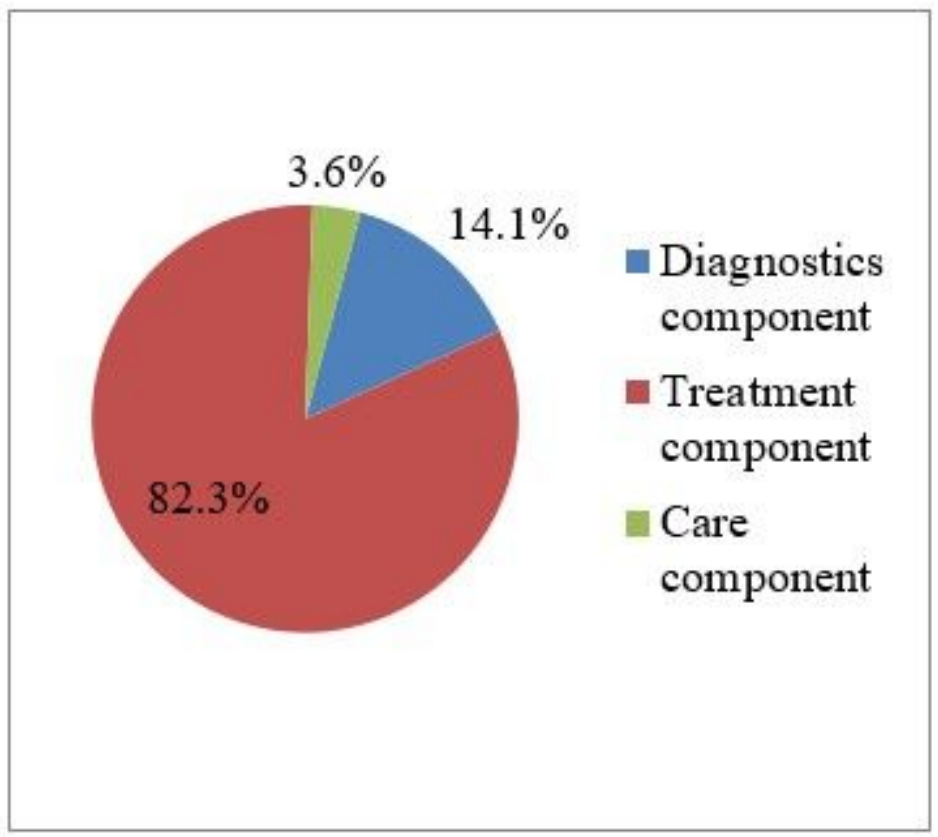

Figure 2

Main components of hospitalization expenditure 


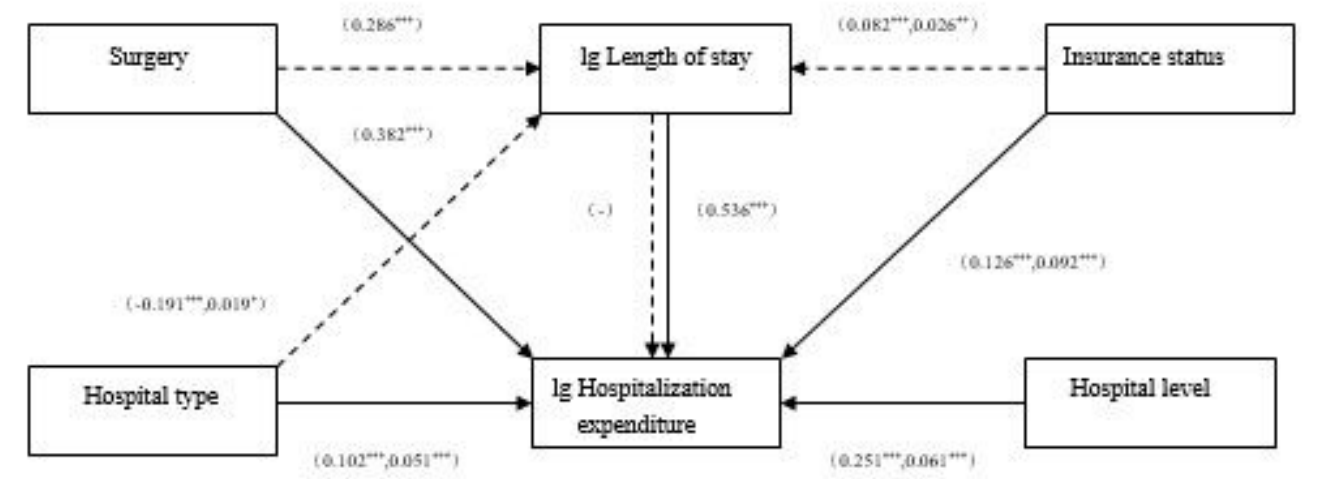

Figure 3

Path analysis diagram Note: ${ }^{\star} P<0.05 .{ }^{*} P<0.01$. ${ }^{* *} P<0.001$. The numbers in brackets are the standardized estimate $(\beta)$ for dummy variables.
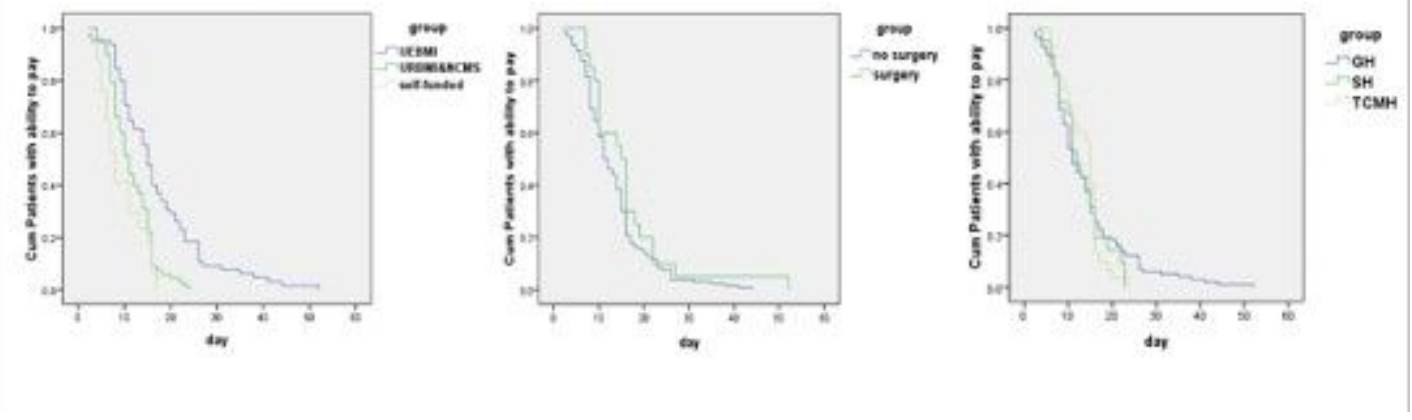

\section{Figure 4}

Survival analysis plot 